\title{
Wearables, Physical Activity, and Exercise Testing in Liver Disease
}

\author{
Michael A. Dunn, MD ${ }^{1,2}$ Matthew R. Kappus, MD ${ }^{3}$ Pamela M. Bloomer, PT, MPT ${ }^{1,2}$ \\ Andres Duarte-Rojo, MD, $\mathrm{PhD}^{1,2}$ Deborah A. Josbeno, $\mathrm{PT}, \mathrm{PhD}^{4}$ John M. Jakicic, $\mathrm{PhD}^{5}$ \\ ${ }^{1}$ Center for Liver Diseases, Thomas E. Starzl Transplantation Institute, \\ University of Pittsburgh, Pittsburgh, Pennsylvania \\ 2 Pittsburgh Liver Research Center, University of Pittsburgh, \\ Pittsburgh, Pennsylvania \\ ${ }^{3}$ Gastroenterology Division, Department of Medicine, Duke \\ University School of Medicine, Durham, North Carolina \\ ${ }^{4}$ Department of Physical Therapy, School of Health and Rehabilitation \\ Sciences, University of Pittsburgh, Pittsburgh, Pennsylvania \\ ${ }^{5}$ Healthy Lifesty le Institute, University of Pittsburgh, Pittsburgh, \\ Pennsylvania

\begin{abstract}
Address for correspondence Michael A. Dunn, MD, Center for Liver Diseases, Thomas E. Starzl Transplantation Institute, University of Pittsburgh, Kaufmann 201, 3459 Fifth Avenue, Pittsburgh, PA 15213 (e-mail: mad206@pitt.edu).
\end{abstract} \\ Semin Liver Dis 2021;41:128-135.
}

\begin{abstract}
Physical inactivity is a major cause of deterioration in all forms of advanced liver disease. It is especially important as a driver of the components of the metabolic syndrome, with nonalcoholic fatty liver disease rapidly becoming the dominant cause of liver-related death worldwide. Growing realization of the health benefits of moderate-to-vigorous physical activity has captured the interest of persons who desire to improve their health, including those at risk for chronic liver injury. They are

Keywords

- physical activity trackers

- cardiopulmonary exercise testing

- cirrhosis

- liver transplantation increasingly adopting wearable activity trackers to measure the activity that they seek to improve. Improved physical activity is the key lifestyle behavior that can improve cardiorespiratory fitness, which is most accurately measured with cardiopulmonary exercise testing (CPET). CPET is showing promise to identify risk and predict outcomes in transplant hepatology. Team effort among engaged patients, social support networks, and clinicians supported by web-based connectivity is needed to fully exploit the benefits of physical activity tracking.
\end{abstract}

Physical activity is likely the least emphasized, least practiced, but most beneficial intervention known for patients with many forms of chronic liver disease. Inactivity is well recognized as a central driver of all the elements of the metabolic syndrome. With the rapid growth of nonalcoholic fatty liver disease and nonalcoholic steatohepatitis (NASH) as the most prevalent cause of serious liver disease worldwide, improved physical activity has become a central component of therapy for this predominant liver disease and the other elements of the metabolic syndrome. ${ }^{1}$

It seems clear that persons with a chronic illness driven by lack of physical activity who appreciate its potential for improvement would desire to increase their activity levels.
Indeed, patients with advanced liver cirrhosis preparing for liver transplantation almost universally report that they consider exercise to be an important element of their care. ${ }^{2}$ While they recognize the concept, it is a challenge to convert that recognition into action. For example, there is a major gap between cirrhotic patients' self-assessed physical performance and their actual activity as measured by objective monitoring; in terms of steps per day they are among the most sedentary of populations, comparable to persons with end-stage cardiac, pulmonary, or renal disease. ${ }^{3}$

While the concept of inactivity as detrimental and, conversely, exercise as beneficial in NASH and NASH cirrhosis is a worthwhile area of study, it is new and relatively 
unexplored compared with exercise in heart disease: from 1980 to now, there have been 15,345 PubMed citations for "exercise and heart failure" compared with 677 for "exercise and liver cirrhosis." There were 2,696 citations for "exercise and heart transplant" compared with 496 for "exercise and liver transplant."

Objective monitoring of physical activity with wearable technology has intrinsic appeal as a way to incorporate selfregulation in this important lifestyle behavior that will raise self-awareness, empower self-improvement, and, with shared information, partner with caregivers and social support networks. In this review, we will report the current knowledge and assess potential opportunities for using wearable technology and objective exercise testing to guide management to improve the health of patients with chronic liver disease and cirrhosis.

\section{Wearable Activity Trackers}

A personal activity tracker is a device designed to be worn on the user's body that uses accelerometers, altimeters, gyroscopes, thermal flux, or other sensors to track the wearer's movements, heart rate, and biometric data, and uploads the data to an online application that shows trends over time. ${ }^{4}$

Their use is consistent with and supports growing interest in the "quantified self," a concept that holds that any aspect of human activity that must be improved has to be measured to improve it. ${ }^{5}$ From tracking running pace and distances, through the use of pocket or watch-based pedometers for over 200 years, to the present-day consumer electronics revolution, there has been an exponential growth in accuracy, functionality, and ease of wear of these devices, along with their steadily increasing uptake. Annual global revenue for fitness trackers is $\$ 2.5$ billion, with 65 million annual shipments of wearable wristband devices. ${ }^{6}$ In the United States according to a 2015 Centers for Disease Control (CDC) study, one in four adults has used a personal activity tracker, and one in eight reports current regular use, with a strong bias for use by more active individuals: $17 \%$ of persons who self-report activity that meets CDC's recommended level of $\geq 150 \mathrm{~min} /$ week of moderate-to-vigorous activity regularly use a tracker, versus $4 \%$ of inactive individuals. ${ }^{7}$

The observation that active persons who place high value on the benefit of physical activity tend to use trackers on a sustained basis was confirmed by focus group insights provided by older adults. They noted that ease of use, ability to self-monitor performance, and most importantly, appreciation and enthusiasm for the social value of comparing their activity with that of friends or family were their key motivators for sustained use. ${ }^{8}$ On the other hand, an important unresolved issue that limits the value of activity tracker information for improving health is lack of provider awareness of measured activity or lack of incorporation of the information into a plan of care. Only a minority of regular physical activity tracker users say that they have shared the information with their providers. ${ }^{5}$ When performance data are not incorporated into electronic records in a longitudinally displayed, actionable manner, the information is less likely to trigger appropriate responses by a care team. ${ }^{9}$ The care of patients with advanced liver diseases is understandably driven by data, such as the results of laboratory testing, vital signs, imaging, and endoscopic findings that elicit comments and actions. While we now appreciate the central importance of physical activity in the management of NASH and NASH cirrhosis, we lack a systematic way to appreciate and respond to activity changes in the same way that we react to other key data: for example, a review of a sample of 50 consecutive visit notes for patients with NASH in our center showed that only six notes included a comment about physical activity.

\section{Activity Measurements}

-Fig. 1 shows the categories of wearable activity monitors that may be useful for patients with liver disease. Beginning in 1780 with the capability to record daily step counts and distances walked using watch-based pedometers, ${ }^{4}$ devices progressed in recent years to electronic monitors using wearable accelerometers, first single-axis and later triaxial. The trackers subsequently added sensors to detect thermal flux, light-dependent plethysmographic heart rate, and global positioning. Multisensor trackers were developed as research devices that required chest or upper arm placement. Over the past one to two decades, over 400 commercial activity trackers, many of which are worn on the wrist or embedded into a watch, have been marketed by over 100 vendors. User features have included improved wearability, an extended battery life, and Bluetooth connectivity to visually appealing smartphone displays. ${ }^{10}$ Some of these compact devices have also included multisensors that integrate multiple inputs to calculate derivative outputs including movement displayed as steps and distance, exercise intensity in metabolic equivalents (METs), energy expenditure in kilocalories, and sleep duration and quality. Additional devices (Polar, Zephyr harness, Whoop strap) now provide the capability to record variability in heart rate with accuracy similar to that of Holter monitoring.

Recently, smart textiles have been introduced with a capability for physiologic signal monitoring owing to the development of sensing electrodes that can be woven, knitted, or embroidered into clothing. Athletic shirts or other clothing items made with such conductive textiles can now reliably acquire signals to track physical activity by monitoring electromyography in addition to textile-based embedded monitoring of neurologic function using electroencephalography, and cardiac function using electrocardiography (ECG). ${ }^{11}$

The algorithms used for commercially available multisensor activity tracking devices are typically proprietary, closely guarded as trade secrets. This may be problematic for longitudinal health care and research because they can be modified or upgraded without notice to users, which may result in information changing solely based on modifications to these algorithms. Moreover, their development and testing in persons in good general health may limit their applicability to patients with chronic conditions such as liver disease. 


\begin{tabular}{|c|c|c|c|}
\hline Device & Examples & Illustrations & Functionality \\
\hline Pedometers & $\begin{array}{l}\text { Step-activated Swiss } \\
\text { watch (Perrelet, 1780), } \\
\text { Yamasa (Japan 1960s) } \\
\text { Omron, Igank }\end{array}$ & & Distance and steps \\
\hline $\begin{array}{l}\text { Research } \\
\text { trackers }\end{array}$ & $\begin{array}{l}\text { Accelerometers (Caltrac, } \\
\text { TriTrac, Actigraph) and } \\
\text { multisensor devices (e.g., } \\
\text { Sensewear) }\end{array}$ & & $\begin{array}{l}\text { Distance, steps, activity intensity, energy } \\
\text { expenditure } \\
\text { Multiple sensors: triaxial accelerometers, } \\
\text { global positioning, heart rate via } \\
\text { plethysmography, galvanic skin response, } \\
\text { thermal flux }\end{array}$ \\
\hline $\begin{array}{l}\text { Mass market } \\
\text { trackers }\end{array}$ & $\begin{array}{l}423 \text { devices, } 132 \text { brands, } \\
\text { top } 5 \text { include Fitbit, } \\
\text { Garmin, Misfit, Apple, } \\
\text { Polar }\end{array}$ & & $\begin{array}{l}\text { Includes accelerometers and other } \\
\text { sensors, designed for ease of wear (e.g., } \\
\text { wrist worn), data displayed on the } \\
\text { devices, smartphones, or potentially via } \\
\text { web application program interface (API) } \\
\text { to clinical record systems }\end{array}$ \\
\hline $\begin{array}{l}\text { Multicomponent } \\
\text { devices }\end{array}$ & $\begin{array}{l}\text { Zephyr harness, } \\
\text { Polar H10 chest strap, } \\
\text { Whoop strap }\end{array}$ & & $\begin{array}{l}\text { Added capabilities for heart rate } \\
\text { variability and with Whoop for advanced } \\
\text { support of high-performance athletes }\end{array}$ \\
\hline Smart textiles & $\begin{array}{l}\text { Garments with } \\
\text { electrodes woven, } \\
\text { knitted or embroidered } \\
\text { into textiles for } \\
\text { physiologic functions } \\
\text { such as EMG muscle } \\
\text { status monitoring }\end{array}$ & & $\begin{array}{l}\text { The blue dots indicate positions of } \\
\text { embedded textile electrodes }\end{array}$ \\
\hline
\end{tabular}

Fig. 1 Physical activity trackers. The development, appearance, and functionality of various classes of physical activity trackers are illustrated. Details and references are provided in the Activity Measurements section of the accompanying text.

\section{Daily Steps}

As shown in - Fig. 1, for nearly 250 years, there have been attempts to measure steps and distance traveled per day. The common concept that 10,000 steps/day is useful to sustain health in normal adults is supported by some studies ${ }^{7}$; however, a recent review has reported that adding 2,000 steps per day is associated with health benefits. ${ }^{12}$ Patients with liver cirrhosis are well known to have lower physical activity levels than healthy persons, with considerable variability depending on the extent of liver dysfunction. ${ }^{13} \mathrm{~A}$ count of 5,000 steps/day was proposed as an indicator of clinical stability and low likelihood for occurrence of decompensating complications in a cohort of cirrhotic patients from Japan. ${ }^{14}$ Lower daily step counts were associated with a greater risk of death or decompensation. As noted above, ${ }^{3}$ we found that patients with more advanced liver disease, such as those waitlisted for liver transplantation, had lower daily step counts, 3,200 \pm 2800 , similar to those of patients with advanced cardiac or pulmonary failure or dialysis-dependent renal failure. Self-awareness by cirrhotic patients of their extent of inactivity is often limited; in spite of severe inactivity they subjectively rate their physical capacity as relatively robust when using the Karnofsky Performance and Rosow-Breslau scales.

Two pilot studies of home-based exercise in cirrhotic patients involved activity tracker monitoring. Williams et al showed improvement in average daily steps from a mean of 4,000 at baseline to 6,700 after a 12 -week intervention in 18 transplant candidates. ${ }^{15}$ Chen et al reported a randomized pilot trial of 17 cirrhotic patients, with 9 completing a 12-week home exercise program and 8 usual care subjects; the intervention patients increased their mean daily step count from 4,500 to 5,600 /day, while the step count for control patients decreased from 4,400 to 2,900/day, a significant difference favoring the intervention group. ${ }^{16}$

\section{Activity Intensity}

We measured activity intensity with a multifunctional BodyMedia SenseWear armband (Jawbone Inc.) in our cohort study of 53 transplant waitlisted patients cited above. Their 
average waking time spent in sedentary activity ( $<1.5$ METs) was $76 \%$ compared with $62 \%$ for an elderly reference population, and their percentage of moderate-to-vigorous activity ( $\geq 3$ METs) was $5 \%$ compared with $16 \%$ for the reference population, both highly significant. There was a positive association of sedentary activity and a negative association of moderate-to-vigorous activity with the nine waitlist deaths in the cohort. ${ }^{3}$

\section{Heart Rate Variability}

Healthy persons with normal cardiac rhythm show heart rate variability (HRV) that reflects a balance of sympathetic and parasympathetic autonomic influences. ${ }^{17}$ Autonomic dysfunction commonly suppresses HRV; decreased HRV is a feature of decompensated cirrhosis and states of systemic inflammation, such as cardiac ischemia, sepsis, and diabetes. A common measurement of HRV is the standard deviation of all normal RR intervals between consecutive sinus beats, SDNN, measured by analysis of Holter monitor records. Normal SDNN values, on the order of 50 milliseconds, decrease with age. As shown in - Fig. 1, some activity monitoring devices can report HRV as SDNN values. Four reports show that lower HRV measured by wearable monitoring or ECG was associated with liver transplant waitlist mortality, ${ }^{17}$ mortality in advanced cirrhosis, ${ }^{18}$ degree of hepatic encephalopathy, ${ }^{19}$ and risk for acute-on-chronic liver failure and death. ${ }^{20} \mathrm{~A}$ limitation of the clinical utility of $\mathrm{HRV}$ is that $\beta$ blockers, often administered to patients with cirrhosis, suppress HRV.

\section{Cadence}

Step count, the oldest measurement of daily physical activity, continues to have merit as an activity index as noted above, is independent of proprietary algorithms, and remains valid in the presence of autonomic medications such as $\beta$ blockers or rhythm disturbances. We explored whether per-minute steps, or cadence, could have value as a simple, reliable activity intensity metric as described earlier. ${ }^{21}$ We evaluated cadence in our 17-patient pilot exercise intensity study described above ${ }^{16}$ in which we asked the nine patients performing home-based exercise to walk at a cadence of 100 steps/min for $30 \mathrm{~min} /$ day, versus usual activity in eight. ${ }^{22}$ We evaluated cadence by analyzing over 2.25 million tracker-monitored minutes, and compared the changes in intensity shown by cadence with two other measurements: (1) intensity in METs using the proprietary Fitbit algorithm (Fitbit, San Francisco, CA) and (2) heart rate reserve (HRR) calculated according to the American College of Sports Medicine's exercise testing guideline. ${ }^{23}$ We found that cadence paralleled changes in maximum and minimum activity in steps/day more accurately and consistently than did either Fitbit-reported intensity or HRR. ${ }^{22}$ We illustrate a further analysis of these reported data in - Fig. 2. It shows that considering the differences in measuring scales, patients were sedentary 88 to $95 \%$ of study time and performed moderate-to-vigorous activity for 8 to $42 \mathrm{~min} / \mathrm{day}$, which represented $<1 \%$ of the day for cadence, $2 \%$ for Fitbit METs, and $3 \%$ for HRR. Interestingly, in 39\% of the minutes classified as ranging from moderate to maximal intensity based on

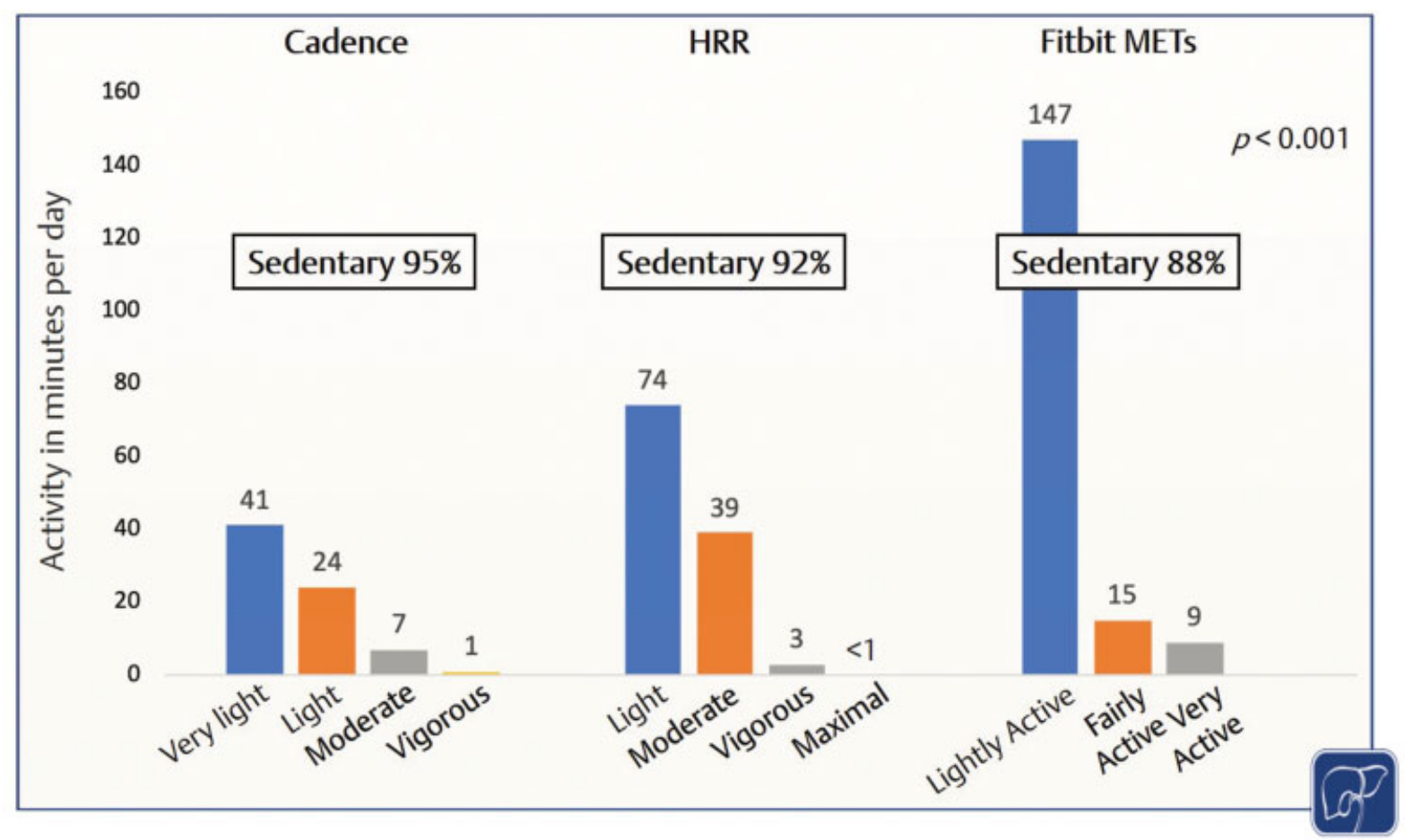

Fig. 2 Number of daily minutes spent at each activity category according to three measuring methods. The minutes spent at the lowest intensity (i.e., sedentary) are shown as percentage of 1,440 minutes/day. Cadence categories: sedentary 0-19 steps/min, very light 20-39, light 40-79, moderate 80-130, and vigorous $>130$. Heart rate reserve (HRR) categories: very light activity $<30 \%$, light $30-39$, moderate $40-59$, vigorous 60-89, and maximal $\geq 90$. Fitbit metabolic equivalent (MET) categories: sedentary $<1.5 \mathrm{METs}$, lightly active $1.5-3$, fairly active $3-6$ for $\geq 10$ minutes, and very active $\geq 6$ for $\geq 10$ minutes. Comparison was performed combining very light plus light activities in the same category for cadence, and vigorous plus maximal for HRR. 
HRR, the accelerometer registered 0 steps, showing either an elevated baseline heart rate without accompanying physical activity or perhaps activity without translational movement, such as stationary cycling. Measurements relying on heart rate alone as a biomarker of exercise in cirrhosis may overestimate activity if not linked to another physiological or activity measurement. We conclude based on these data that the potential value of cadence as an alternative activity measurement merits further exploration.

\section{Cardiopulmonary Exercise Testing in Liver Disease}

Wearable monitors are intended to assist users to measure and help promote performance of physical activity over time, with a goal of improving the user's stamina, resilience, and physical capacity to withstand stress. Measuring the capacity for maximum physical performance is an important complementary fitness metric that involves testing users' ability to generate maximum effort during cardiopulmonary exercise to show the intensity of activities a patient can tolerate, to determine if a physical activity program is sufficient to improve cardiopulmonary capacity, and to indicate health risk.

Cardiopulmonary exercise testing (CPET) is a noninvasive, accurate method that represents the gold standard to quantitate exercise capacity and physical functional reserve. ${ }^{24} \mathrm{It}$ measures aerobic capacity, the body's ability to consume and use oxygen during exercise. ${ }^{25,26}$ Aerobic capacity reflects the integration of pulmonary, cardiovascular, hematopoietic, neuropsychological, and skeletal muscle performances beyond measurement of the capacity of any single organ system. CPET is a useful tool for understanding responses to stress in patients with cirrhosis, who frequently have impaired maximal $\left(\mathrm{VO}_{2}\right.$ max $)$ or peak $\left(\mathrm{VO}_{2}\right.$ peak $)$ oxygen utilization, indicating reduced aerobic capacity.

CPET has attracted clinical interest beyond its initial use as a research tool in exercise physiology to engage a spectrum of medical providers who seek to better understand the significance of maximal or submaximal/peak exercise responses. While initially explored for preoperative risk stratification for cardiopulmonary surgery, CPET is now increasingly used as a stratification tool in noncardiopulmonary surgery. A systematic review showed that CPET variables outperformed alternative methods of preoperative risk stratification for noncardiopulmonary major procedures. ${ }^{27}$

In liver disease, CPET was reported to be a strong predictor of major postoperative morbidity for 104 patients undergoing elective hepatic resection. ${ }^{28}$ In 27 patients with cirrhosis, $\mathrm{VO}_{2}$ max was inversely correlated with the Model for EndStage Liver Disease (MELD) score $(r=-0.91)$. Those with a $\mathrm{VO}_{2 \max }<14 \mathrm{~mL} / \mathrm{kg} / \mathrm{min}$ had a highly significant increased risk of 3-year mortality (odds ratio of 3.2). ${ }^{29} \mathrm{~A}$ single-center study that evaluated aerobic capacity with CPET in 399 potential liver-transplant candidates showed that both $\mathrm{VO}_{2}$ peak and anaerobic threshold (AT), the point at which a shift from aerobic to anaerobic metabolism occurs, were lower in patients who did not survive to liver transplant. ${ }^{30}$
CPET helps to predict survival after liver transplantation. In patients who survived 90 days after transplant, the mean AT was significantly higher in survivors than in nonsurvivors. ${ }^{31}$ In a 60-patient cohort who underwent pretransplant CPET testing, only AT was retained as a significant predictor of posttransplant mortality. Receiver operating characteristic curve analysis showed sensitivity and specificity of 90.7 and $83.3 \%$, respectively, with good model accuracy (AUROC $=0.92$; $95 \%$ confidence interval [CI]: 0.82-0.97; $p=0.001$ ). Ney et al performed a systematic review of pre- and posttransplant mortality in seven published studies that included 1,107 patients at a mean MELD of 14.2 and a mean baseline $\mathrm{VO}_{2 \text { peak }}$ of $17.4 \mathrm{~mL} / \mathrm{kg} /$ min. ${ }^{32}$ CPETvariables were independent predictors of pretransplant mortality (three studies) and posttransplant mortality (four studies). For perspective, a $\mathrm{VO}_{2}$ max of $17.4 \mathrm{~mL} / \mathrm{kg} / \mathrm{min}$ corresponds to the cardiopulmonary fitness of persons 3 decades older than observed and below the threshold for full independent living. ${ }^{33}$

CPET also serves as a reference standard to measure the benefit of physical training regimens in patients with cirrhosis, in whom $\mathrm{VO}_{2}$ peak may improve by up to $4.5 \mathrm{~mL} / \mathrm{kg} / \mathrm{min}$ after 8 to 16 weeks of an exercise intervention, which is a clinically relevant effect expected to positively impact on mortality. ${ }^{34-36}$ CPET allows a physiological determination of maximum effort when the respiratory exchange ratio is $>1.1$, a relevant feature that other methods fail to show. This threshold is not reached by approximately $20 \%$ of patients with decompensated cirrhosis. ${ }^{16}$ In patients who do not achieve the threshold, the ventilatory efficiency slope $\left(\mathrm{VE} / \mathrm{VCO}_{2}\right.$ ) can be used instead of $\mathrm{VO}_{2 \text { peak }}$ to assess improvement in cardiopulmonary fitness as a response to exercise training. ${ }^{37}$ In our experience, when considering either $\mathrm{VO}_{2 \text { peak }}$ or $\mathrm{VE} / \mathrm{VCO}_{2}$, over $60 \%$ of decompensated patients have a high cardiovascular risk as determined by $\mathrm{VO}_{2}$ peak $\leq$ $14 \mathrm{~mL} / \mathrm{kg} / \mathrm{min}$ or $\mathrm{VE} / \mathrm{VCO}_{2}>36$, respectively. ${ }^{16}$

To conclude, CPET can identify impaired exercise capacity as a biomarker of pre- and post-liver transplant mortality risk and assess the benefit of exercise interventions. CPET performance merits exploration as an adjunctive parameter for organ allocation or transplant listing decisions, as well as for identification of patients who would benefit from intensified dietary, physical activity, or pharmacologic interventions. Disadvantages of CPET include its time and resource costs and its requirement for expert performance and data interpretation. CPET can, however, provide an objective reference point to learn whether and to what extent aerobic capacity will prove to be a modifiable risk in patients with advanced liver disease.

\section{Practical Challenges in Promoting Physical Activity in Patients with Cirrhosis: Perspective of a Physical Therapist}

Encouraging monitoring of physical activity with wearable trackers has been a valuable way to support home-based exercise for our liver-transplant candidates since we began their use in 2016. As we review the situation today, however, the present COVID-19 pandemic has shifted our emphasis for 
prehabilitation from in-person clinic visits with hands-on testing and coaching to telehealth encounters with greater reliance on information from trackers. The shift is a challenge because it is at odds with therapists' traditional training that emphasizes hands-on experience and in-person functional testing to assess patients' success in reaching and adjusting performance goals.

Compliance with tracker use has never been ideal, but it has now become especially important because patients confined to their homes lack access to the usual resources-fitness facilities, pools, malls, and physical therapy clinics-that normally support activity. Physical therapists and clinical exercise physiologists who can remotely monitor tracker data can see gaps caused by nonuse, user error, or technology breakdowns. In fact, experience treating other health conditions such as obesity shows the effectiveness of combining activity tracking with health coaching from an appropriately trained professional. ${ }^{38,39}$ When gaps occur that arise from nonuse or decreased use over time, ${ }^{40}$ health professionals can engage patients to assess barriers to use and use voice or video technology to help them generate solutions to improve compliance.

Patients with cirrhosis who are older and have never used an activity tracker are more likely to be successful if they can rely on a robust infrastructure of both human and technical support. They need their own or a caregiver's ability to use key features of a smartphone, as well as clear, simple written guidance on keeping the device charged, clean and functional, and ensuring it maintains internet connectivity. Patients need an easily accessible, responsive contact in the clinic who can resolve technical issues. The best assurance that a patient will be able to use a tracker to sustain improved activity is their having an interested, supportive family member or friend who understands tracker use and has the commitment to help the patient.

Given the growing proliferation of many trackers with multiple features, a one-size-fits-all tracker prescription seems unlikely to benefit all patients. We recommend that patients, guided by a physical therapist or other exercise professional, begin with a tracker that best seems to fit their needs and level of interest in its specific features, and base their continued use versus opting to change to a different device on their personal satisfaction and their level of performance.

In our anecdotal experience, COVID-19-mandated social distancing appears to intensify patients' intrinsic predisposition for use or nonuse of trackers: patients who do not want to wear the tracker may feel that they cannot be as mobile or as active as they were before and therefore see no benefit in wearing it. However, after a check-in call to reinforce and explain the importance of wearing the tracker and the value of staying active during this time more than ever to remain strong, they will frequently agree and see the tracker in the same light as the physical therapist. There will be an increase in compliance, at least temporarily, until the cycle begins again necessitating another call. If a data gap is due to a true technology issue, we attempt to walk the patient through the steps of reconnection via phone or video. Motivation again is the key driver: reconnection is much easier in more active versus more sedentary users, so that we have confirmed from this experience that patients who are more active at baseline are far more likely to use, benefit from, and improve their activity with trackers under social distancing constraints than those with sedentary activity at baseline. Conversely, patients who are not as active often lack the desire to reconnect and fix technical problems, rendering the tracker ineffective.

We believe that an unexpected decrease in physical activity level may be the first signal that heralds an unexpected major complication of cirrhosis, such as a bout of encephalopathy, infection, or perturbation in fluid balance. We think that as more cirrhotic patients make longitudinal use of trackers, their potential value to indicate the onset of decompensating events will become more clear.

At our center, we provide patients with trackers owned by the institution. We encourage patients to self-monitor their activity levels with data displayed on personal smartphones. We track and monitor patients' physical performance using a web-based, clinical security compliant and privacy-protected application program interface linking the tracker to a secure data store within our electronic clinical domain. At present, patients using the trackers agree not to connect them to commercial user groups or communities that have the potential to share activity information without assured privacy and security. At present, the use of trackers and their data for patient care in the United States has not been identified as an area requiring their government oversight or regulation as a medical device.

We recognize the value of user community social support and gamification (the application of typical beneficial elements of group game play) for promoting activity. We are developing an integrated multifunctional instructional, activity, nutritional support, gamification, and tracking application for patients with end-stage liver disease consistent with appropriate privacy and security, El-Fit, funded in part by a pilot grant to one of us (A.D-.R.) from the American Association for the Study of Liver Diseases and described at https://upitt. resoluteinnovation.com/technologies/04956_el-fit-exerciseand-liver-fitness.

\section{Conclusion}

We have come to appreciate that a cardinal manifestation of advanced liver disease is physical decline, associated with inactivity and manifested as the phenotype of frailty, diminished capacity to withstand physical stress. ${ }^{41}$ Frailty, a leading and potentially reversible consequence of inactivity and cause of liver transplant waitlist mortality, ${ }^{42}$ must be accurately measured with tools such as the Liver Frailty Index, a test that predicts adverse outcomes in patients with advanced cirrhosis. ${ }^{43}$

Physical activity is now recognized as an important part of the prescription to improve the health and prognosis of persons with advanced liver disease, especially those awaiting transplantation. ${ }^{44}$ After transplantation, physical activity remains essential for promoting full recovery of physical function, maintaining metabolic health, and achieving satisfying quality of life. ${ }^{45}$ Wearables and CPET are our most promising tools to 
assess baseline physical activity and cardiopulmonary capacity, respectively, and to monitor the efficacy of interventions, especially when they leverage opportunities that web-based data-sharing wearables provide for social networking support and provider engagement.

As yet, very little is understood about the most effective proportions of light and moderate-to-vigorous intensity activity, and of the potential benefits of different forms of physical activity (e.g., aerobic and resistance training) that would best promote desirable outcomes in patients with advanced liver disease. ${ }^{46}$ Patients who desire empowerment to take charge of their own health and who appreciate that what needs to be improved needs to be measured with selfmonitoring hold the potential to become a strong force for effective use of wearable activity tracking in hepatology.

\section{Main Concepts and Learning Points}

- Physical inactivity is a key driver of progression and adverse outcomes in advanced liver diseases, especially nonalcoholic fatty liver disease, the dominant chronic liver disease today.

- Wearable personal activity trackers provide patients with a tool for self-monitoring and self-improvement as well as data sharing with providers.

- Cardiopulmonary exercise testing is a parallel method to assess physical capacity in advanced liver disease.

- Web-based data sharing and peer social support offer an opportunity to improve activity.

\section{Conflict of Interest}

Dr. Jakicic reports personal fees from WW International, Inc., outside the submitted work. Dr. Duarte-Rojo reports personal fees from Axcella Health, personal fees from Mallinckrodt, grants and nonfinancial support from Echosens, outside the submitted work.

\section{Acknowledgments}

This work was performed without financial assistance and the authors declare no conflicts of interest.

\section{References}

1 Younossi ZM, Loomba R, Rinella ME, et al. Current and future therapeutic regimens for nonalcoholic fatty liver disease and nonalcoholic steatohepatitis. Hepatology 2018;68(01):361-371

2 Chascsa DM, Lai JC, Dunn MA, et al. Patient and caregiver attitudes and practices of exercise in candidates listed for liver transplantation. Dig Dis Sci 2018;63(12):3290-3296

3 Dunn MA, Josbeno DA, Schmotzer AR, et al. The gap between clinically assessed physical performance and objective physical activity in liver transplant candidates. Liver Transpl 2016;22(10): 1324-1332

4 Macmanus R. Health Trackers: How Technology Is Helping Us to Monitor and Improve Our Health. Lanham, MD: Rowman and Littlefield; 2015

5 Hoy MB. Personal activity trackers and the quantified self. Med Ref Serv Q 2016;35(01):94-100

6 Statista. Wearable technology - statistics \& facts. Available at: https://statista.com/topics/1556/wearable-technology. Accessed May 8, 2020
7 Omura JD, Carlson SA, Paul P, Watson KB, Fulton JE. National physical activity surveillance: users of wearable activity monitors as a potential data source. Prev Med Rep 2016;5:124-126

8 Kononova A, Li L, Kamp K, et al. The use of wearable activity trackers among older adults: focus group study of tracker perceptions, motivators, and barriers in the maintenance stage of behavior change. JMIR Mhealth Uhealth 2019;7(04):e9832

9 Chung CF, Cook J, Bales E, Zia J, Munson SA. More than telemonitoring: health provider use and nonuse of life-log data in irritable bowel syndrome and weight management. J Med Internet Res 2015;17(08):e203

10 Henriksen A, Haugen Mikalsen M, Woldaregay AZ, et al. Using fitness trackers and smartwatches to measure physical activity in research: analysis of consumer wrist-worn wearables. J Med Internet Res 2018;20(03): 1110

11 Acar G, Ozturk O, Golparvar AJ, et al. Wearable and flexible textile electrodes for biopotential signal monitoring: a review. Electronics (Basel) 2019;8:479

12 Kraus WE, Janz KF, Powell KE, et al; 2018 PHYSICAL ACTIVITY GUIDELINES ADVISORY COMMITTEE*. Daily step counts for measuring physical activity exposure and its relation to health. Med Sci Sports Exerc 2019;51(06):1206-1212

13 Pattullo V, Duarte-Rojo A, Soliman W, et al. A 24-week dietary and physical activity lifestyle intervention reduces hepatic insulin resistance in the obese with chronic hepatitis C. Liver Int 2013;33 (03):410-419

14 Hayashi F, Momoki C, Yuikawa M, et al. Nutritional status in relation to lifestyle in patients with compensated viral cirrhosis. World J Gastroenterol 2012;18(40):5759-5770

15 Williams FR, Vallance A, Faulkner T, et al. Home-based exercise in patients awaiting liver transplantation: a feasibility study. Liver Transpl 2019;25(07):995-1006

16 Chen HW, Ferrando A, White MG, et al. Home-based physical activity and diet intervention to improve physical function in advanced liver disease: a randomized pilot trial. Dig Dis Sci 2020; 65(11):329-335

17 Fleisher LA, Fleckenstein JF, Frank SM, Thuluvath PJ. Heart rate variability as a predictor of autonomic dysfunction in patients awaiting liver transplantation. Dig Dis Sci 2000;45(02): 340-344

18 Ates F, Topal E, Kosar F, et al. The relationship of heart rate variability with severity and prognosis of cirrhosis. Dig Dis Sci 2006;51(09):1614-1618

19 Mani AR, Montagnese S, Jackson CD, et al. Decreased heart rate variability in patients with cirrhosis relates to the presence and degree of hepatic encephalopathy. Am J Physiol Gastrointest Liver Physiol 2009;296(02):G330-G338

20 Jansen C, Chatterjee DA, Thomsen KL, et al. Significant reduction in heart rate variability is a feature of acute decompensation of cirrhosis and predicts 90-day mortality. Aliment Pharmacol Ther 2019;50(05):568-579

21 Tudor-Locke C, Han H, Aguiar EJ, et al. How fast is fast enough? Walking cadence (steps/min) as a practical estimate of intensity in adults: a narrative review. Br J Sports Med 2018;52(12): 776-788

22 Chen HW, Ferrando AA, Dunn MA, Kim WR, Duarte-Rojo A. Cadence from physical activity trackers for monitoring of home-based exercise intensity in advanced liver disease. Liver Transpl 2020;26(05):718-721

23 Riebe D, Ehrman JK, Liguori G, Magel M. ACSM's Guidelines for Exercise Testing and Prescription. 10th ed Philadelphia, PA: Wolters Kluwer; 2018

24 American Thoracic Society American College of Chest Physicians. ATS/ACCP statement on cardiopulmonary exercise testing. Am J Respir Crit Care Med 2003;167(02):211-277

25 Lemyze M, Dharancy S, Wallaert B. Response to exercise in patients with liver cirrhosis: implications for liver transplantation. Dig Liver Dis 2013;45(05):362-366 
26 Oudiz RJ, Barst RJ, Hansen JE, et al. Cardiopulmonary exercise testing and six-minute walk correlations in pulmonary arterial hypertension. Am J Cardiol 2006;97(01):123-126

27 Hennis PJ, Meale PM, Grocott MP. Cardiopulmonary exercise testing for the evaluation of perioperative risk in non-cardiopulmonary surgery. Postgrad Med J 2011;87(1030):550-557

28 Junejo MA, Mason JM, Sheen AJ, et al. Cardiopulmonary exercise testing for preoperative risk assessment before hepatic resection. Br J Surg 2012;99(08):1097-1104

29 Galant LH, Forgiarini Junior LA, Dias AS, Marroni CA. Maximum oxygen consumption predicts mortality in patients with alcoholic cirrhosis. Hepatogastroenterology 2013;60(125):1127-1130

30 Bernal W, Martin-Mateos R, Lipcsey M, et al. Aerobic capacity during cardiopulmonary exercise testing and survival with and without liver transplantation for patients with chronic liver disease. Liver Transpl 2014;20(01):54-62

31 Prentis JM, Manas DM, Trenell MI, Hudson M, Jones DJ, Snowden CP. Submaximal cardiopulmonary exercise testing predicts $90-$ day survival after liver transplantation. Liver Transpl 2012;18 (02):152-159

32 Ney M, Haykowsky MJ, Vandermeer B, Shah A, Ow M, Tandon P. Systematic review: pre- and post-operative prognostic value of cardiopulmonary exercise testing in liver transplant candidates. Aliment Pharmacol Ther 2016;44(08):796-806

33 Forman DE, Arena R, Boxer R. American Heart Association Council on Clinical Cardiology; Council on Cardiovascular and Stroke Nursing; Council on Quality of Care and Outcomes Research; and Stroke Council, et al. Prioritizing functional capacity as a principal end point for therapies oriented to older adults with cardiovascular disease: a scientific statement for healthcare professionals from the American Heart Association. Circulation 2017; 135(16):e894-e918

34 Zenith L, Meena N, Ramadi A, et al. Eight weeks of exercise training increases aerobic capacity and muscle mass and reduces fatigue in patients with cirrhosis. Clin Gastroenterol Hepatol 2014;12(11):1920.e2-1926.e2

35 Debette-Gratien M, Tabouret T, Antonini M-T, et al. Personalized adapted physical activity before liver transplantation: acceptability and results. Transplantation 2015;99(01):145-150
36 Kruger C, McNeely ML, Bailey RJ, et al. Home exercise training improves exercise capacity in cirrhosis patients: role of exercise adherence. Sci Rep 2018;8(01):99

37 Macías-Rodríguez RU, Ilarraza-Lomelí H, Ruiz-Margáin A, et al. Changes in hepatic venous pressure gradient induced by physical exercise in cirrhosis: results of a pilot randomized open clinical trial. Clin Transl Gastroenterol 2016;7(07):e180

38 Rogers RJ, Lang W, Barone Gibbs B, et al. Applying a technologybased system for weight loss in adults with obesity. Obes Sci Pract 2016;2(01):3-12

39 Pellegrini CA, Verba SD, Otto AD, Helsel DL, Davis KK, Jakicic JM. The comparison of a technology-based system and an in-person behavioral weight loss intervention. Obesity (Silver Spring) 2012; 20(02):356-363

40 Finkelstein EA, Haaland BA, Bilger M, et al. Effectiveness of activity trackers with and without incentives to increase physical activity (TRIPPA): a randomised controlled trial. Lancet Diabetes Endocrinol 2016;4:983-995

41 Fried LP, Tangen CM, Walston JCardiovascular Health Study Collaborative Research Group, et al. Frailty in older adults: evidence for a phenotype. J Gerontol A Biol Sci Med Sci 2001;56(03): M146-M156

42 Lai JC, Feng S, Terrault NA, Lizaola B, Hayssen H, Covinsky K. Frailty predicts waitlist mortality in liver transplant candidates. Am J Transplant 2014;14(08):1870-1879

43 Lai JC, Covinsky KE, Dodge JL, et al. Development of a novel frailty index to predict mortality in patients with end-stage liver disease. Hepatology 2017;66(02):564-574

44 Duarte-Rojo A, Ruiz-Margáin A, Montaño-Loza AJ, Macías-Rodríguez RU, Ferrando A, Kim WR. Exercise and physical activity for patients with end-stage liver disease: improving functional status and sarcopenia while on the transplant waiting list. Liver Transpl 2018;24(01):122-139

45 Dunn MA, Rogal SS, Duarte-Rojo A, Lai JC. Physical function, physical activity, and quality of life after liver transplantation. Liver Transpl 2020;26(05):702-708

46 Bellar A, Welch N, Dasarathy S. Exercise and physical activity in cirrhosis: opportunities or perils. J Appl Physiol (1985) 2020;128 (06):1547-1567 